A quasi-distributed temperature sensor interrogated by optical frequency-domain reflectometer

This article has been downloaded from IOPscience. Please scroll down to see the full text article.

2011 Meas. Sci. Technol. 22115204

(http://iopscience.iop.org/0957-0233/22/11/115204)

View the table of contents for this issue, or go to the journal homepage for more

Download details:

IP Address: 193.190.208.37

The article was downloaded on 28/09/2011 at 08:24

Please note that terms and conditions apply. 


\title{
A quasi-distributed temperature sensor interrogated by optical frequency-domain reflectometer
}

\author{
Kivilcim Yüksel, Patrice Mégret and Marc Wuilpart \\ U-MONS, Faculté Polytechnique, Boulevard Dolez 31, 7000 Mons, Belgium \\ E-mail: kivilcim.yuksel@umons.ac.be
}

Received 28 April 2011, in final form 30 August 2011

Published 26 September 2011

Online at stacks.iop.org/MST/22/115204

\begin{abstract}
This paper presents the analysis of a quasi-distributed sensor based on the concatenation of identical low-reflective fiber Bragg gratings. We experimentally demonstrated a temperature sensor using ten cascaded gratings which are interrogated by an optical frequency domain reflectometer. Repeatability measurements highlighted a standard deviation on the measured temperature smaller than $1.5^{\circ} \mathrm{C}$. A complete demonstration of mathematical formulas which are used to obtain the temperature information is also provided.
\end{abstract}

Keywords: fiber optic sensors, fiber Bragg gratings, fiber measurements

(Some figures in this article are in colour only in the electronic version)

\section{Introduction}

Optical fiber optic sensors represent a powerful class of alternative technologies to conventional electrical sensors thanks to their low-weight, small dimensions and immunity to electromagnetic interferences. Among all fiber optic sensors, those based on fiber Bragg gratings (FBGs) have a significant potential. FBGs provide the intrinsic capability of measuring several parameters such as temperature, strain and many others, thanks to its spectral shift as a function of the parameters to be measured. The first commercial FBGs were manufactured in mid-1990s. Since then, in addition to their substantial utilization in the telecommunication industry, parallel efforts have also been carried out to exploit the key features of FBGs for sensing purposes. Nowadays, the sensing sector is benefiting from the great potential of FBG-based sensor systems. Their acceptance is continuously increasing. For sensing applications, FBGs offer the following advantages: they are low-cost, mass producible devices providing self-referencing and wavelength-encoded linear response. Furthermore, these devices allow high degrees of multiplexing (wavelength-, time- or spatial-division), and therefore enable multi-point sensing schemes where many gratings can be placed within a single optical fiber.
Wavelength-division multiplexed (WDM) sensors are the most commercially encountered systems, which permit to address about ten gratings on a single fiber [1]. In these systems, a unique range of operating wavelength (under identical strain and temperature conditions) is dedicated to each grating of the concatenation. Therefore, the number of sensing points is directly limited by the spectral ranges of the source and the detector, and the wavelength spacing between two gratings which depends on the range of the physical parameter to be measured.

In order to overcome these limitations, several solutions based on an optical time domain reflectometer (OTDR) have been proposed [2, 3]. An OTDR launches optical pulses in the fiber under test and detects the backscattered and reflected signals providing the necessary information on position and magnitude along the fiber length, to serve as an interrogating system for quasi-distributed sensors. However, a standard commercial OTDR is not directly suitable to demodulate FBG sensors and is required to be modified into a wavelength-tunable one. Recently, two quasi-distributed temperature sensors based on wavelength-tunable OTDR which interrogates 90 FBGs with $1{ }^{\circ} \mathrm{C}$ of accuracy on the measured temperature were reported [4-6]. The main challenges of these sensors are related to the inevitable deadzone associated with the OTDR detection scheme (which in 
turn limits the minimum physical distance between sensing points), and to the requirement for highly expensive equipment (i.e. tunable OTDR) and quite sophisticated demodulation technique. Moreover, the measurement time needed to interrogate the whole system varies between a few minutes to a few tens of minutes $[4,5]$.

Optical frequency domain reflectometer (OFDR) providing millimeter resolution over medium measurement ranges (up to a few hundreds of meters) has also been attracting great attention as an interrogating tool for several sensor applications [7]. OFDR is a complementary technique to OTDR and provides high spatial resolution possibilities. Instead of working in the time domain as OTDR, OFDR operates in the frequency domain (or Fourier domain). This reflectometry technique can easily be used for the demodulation of FBG reflection spectra. Thanks to the use of a linearly swept optical source, OFDR indeed allows to recover the wavelength reflected by one or several FGBs. The first practical implementation of fiber Bragg grating sensor array interrogated by OFDR was conducted by Froggatt [8] at NASA Langley Research Center and later developed by Luna Innovation of Blacksburg, Virginia [9]. Afterward, the use of FBG arrays interrogated by OFDR for strain sensors was experimentally demonstrated [9-11]. However, the analysis of the operation show some discrepancies in these demonstrations, which can be attributed to differences in definition and unstated differences in simplifying assumptions. Moreover, the spectral-shadowing crosstalk which is inherent to the concatenated FBG array was not evaluated to determine the limitations of the technique.

In this paper, we describe a fiber sensor system for temperature measurement using as sensing points a set of uniform, identical, low-reflective FBGs written in a single fiber and using an OFDR as the interrogator tool. The measurement time is much shorter (a few seconds) than OTDR-based solutions. The measured standard deviation on the temperature is better than $1.5{ }^{\circ} \mathrm{C}$. In addition to experimental work, we provide the full mathematical demonstration of the model used in the analysis. Finally, we evaluate performance limits of our prototype by considering the shadowing effect.

This paper is organized as follows: in section 2 , the principles of the quasi-distributed temperature sensing are explained. Section 3 reports the experimental measurements obtained with a prototype using a concatenation of ten FBGs. The main conclusions of the work are reported in the last section.

\section{Sensor principle}

\subsection{Theoretical background}

The presented sensor is based on the association of two technologies: FBG and OFDR. Gratings act as sensing points whereas the OFDR is used as the interrogating device. In the following paragraphs, these two technologies are briefly introduced.

In its simplest form, a FBG is a permanent and periodic modification of the core refractive index value along an optical fiber. An FBG is described by the following parameters [1]:
- The length $L$ over which the variation on the refractive index is realized. The typical grating length ranges from a few mm up to a few tens of $\mathrm{cm}$.

- The periodicity $\Lambda$ (also called grating pitch) ranges from $200 \mathrm{~nm}$ to $800 \mathrm{~nm}$.

- The amplitude of the refractive index modulation $n$ typically ranges from $10^{-5}$ to $10^{-3}$.

The inscription of such a perturbation in the fiber core refractive index induces mode coupling between two counter propagating beams. That coupling occurs for the resonance wavelength of the grating, the Bragg wavelength, obtained by

$$
\lambda_{\text {Bragg }}=2 n_{\text {eff }} \Lambda,
$$

where $n_{\text {eff }}$ is the effective refractive index of the core at the Bragg wavelength $\lambda_{\text {Bragg }}$.

The FBG acts as a wavelength selective mirror around the Bragg wavelength for which an important reflection is obtained. The Bragg wavelength shift due to a temperature change $\Delta T$ can be written as

$$
\frac{\Delta \lambda_{\text {Bragg }}}{\Delta T}=\lambda_{\text {Bragg }}\left(\frac{1}{n_{\text {eff }}} \frac{\mathrm{d} n_{\text {eff }}}{\mathrm{d} T}+\frac{1}{\Lambda} \frac{\mathrm{d} \Lambda}{\mathrm{d} T}\right) .
$$

- $\frac{1}{n_{\text {eff }}} \frac{\mathrm{d} n_{\text {eff }}}{\mathrm{d} T}$ is the thermo-optic coefficient (i.e. the thermal dependence of the refractive index) and is approximately equal to $8.6 \times 10^{-6} \mathrm{~K}^{-1}$ for germanium doped silica core optical fiber [1].

- $\frac{1}{\Lambda} \frac{\mathrm{d} \Lambda}{\mathrm{d} T}$ is the thermal expansion coefficient of the optical fiber, and relates to the corresponding change in grating periodicity. It is approximately equal to $0.55 \times 10^{-6} \mathrm{~K}^{-1}$ for silica [1].

The refractive index change is the dominant effect and the Bragg wavelength is shifted toward longer wavelengths by temperature. The typical order of magnitude of temperature sensitivity of the Bragg wavelength around $1550 \mathrm{~nm}$ is of $10 \mathrm{pm} /{ }^{\circ} \mathrm{C}$ without spectral shape modification and without hysteresis [1].

OFDR is a technique for high-resolution metrology that can find applications in both optical fiber sensors and telecommunication networks [12].

In its basic configuration, the optical carrier frequency of a tunable laser source (TLS) is swept linearly in time without mode hops. Then, the frequency-modulated continuous-wave signal (probe signal) is split into two paths, namely the test arm and the reference arm (see figure 1). The former includes the device under test (DUT) whereas a reference reflector (also called local oscillator) is placed in the latter. The test signal which is reflected back from the reflection sites in the test arm coherently interfere at the coupler with the reference signal returning from the reference reflector. Superposition of the interfering signals is converted into electrical domain by the detector which yields the beat terms that are related to the optical amplitude and phase responses of the reflection sites in the DUT. In order to observe these beat terms, the photocurrent at the detector output is sampled and Fourier transformed into frequency domain where the beat terms appear as peaks. Using a linear optical frequency sweep, the frequency domain can be mapped into a distance scale 


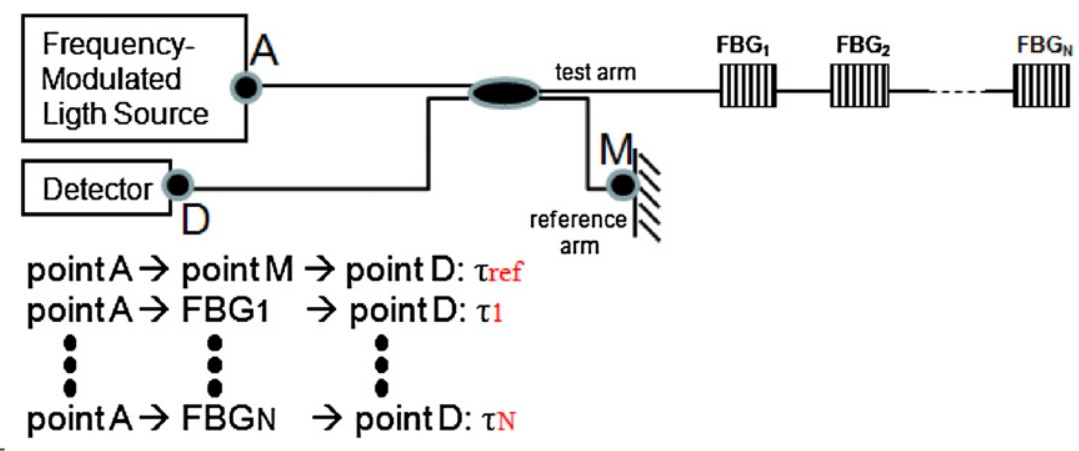

Figure 1. Basic OFDR sensor interrogation set-up with a series of concatenated FBGs.

(the proportionality factor between beat frequency and the corresponding distance is determined by the rate of change of the optical frequency), while the squared magnitude of the signal at each beat frequency reveals the reflectivity of each reflection site.

\subsection{Cascaded FBGs interrogated by OFDR}

Figure 1 shows the block diagram of the OFDR interrogating cascaded FBGs.

As shown in the figure, the probe signal launched into the system is divided into two paths and simultaneously propagates along both the test arm and the reference arm. The total round-trip time of the reference signal's path $(\mathrm{A} \rightarrow \mathrm{M} \rightarrow \mathrm{D})$ is defined as $\tau_{\text {ref }}$ as represented in figure 1 . In the same way, the total round-trip time of the test signal is defined as $\tau_{i}$ for the FBG $\# i$. We start the analysis with the definition of the probe signal which is a continuous wave (CW) optical signal coming from the frequency-modulated light source (point A in figure 1). The complex electric field of the probe signal can be written as

$$
E(t)=E_{0} \exp [\mathrm{j} \phi(t)]
$$

where $E_{0}$ is the amplitude of the electric field and $\phi(t)$ is the phase component. The first derivative of the phase with time is defined as the angular frequency $\omega(t)$,

$$
\omega(t)=\frac{\mathrm{d} \phi(t)}{\mathrm{d} t}
$$

and the phase component can be then written as

$$
\phi(t)=\int_{0}^{t} \omega(u) \mathrm{d} u+\phi_{0},
$$

where $\phi_{0}$ is the initial phase $(t=0)$. For the sawtooth-wave optical frequency modulation case, the optical frequency is swept linearly in time and the angular frequency of the probe signal can be written as

$$
\omega(t)=2 \pi \gamma_{\nu} t+\omega_{0},
$$

where $\omega_{0}$ is the initial angular frequency and $\gamma_{\nu}$ is the tuning rate (in $\mathrm{Hz} \mathrm{s}^{-1}$ ). By using equations (5) and (6), the phase of the probe signal at the TLS output can be written as

$$
\phi(t)=\pi \gamma_{\nu} t^{2}+\omega_{0} t+\phi_{0} .
$$

Therefore, the wavefunction of the probe signal $E(t)$ can be written as

$$
E(t)=E_{0} \exp \left[\mathrm{j}\left(\pi \gamma_{\nu} t^{2}+\omega_{0} t+\phi_{0}\right)\right]
$$

We can now express the optical waves that, after reflection, reach the detector: one, $E_{\text {ref }}(t)$, reflected back from the reference mirror (reference signal) and the other waves, $E_{i}(t)$, reflected from the FBGs (test signals).

The electrical field of the reference signal arriving at the photodedector can be expressed as

$$
\begin{aligned}
& E_{\mathrm{ref}}(t)=\eta_{I L} E\left(t-\tau_{\mathrm{ref}}\right) \exp \left[-\alpha l_{\mathrm{ref}} / 2\right] \\
& =\eta_{I L} E_{0} \exp \left[\mathrm{j}\left(\pi \gamma_{\nu}\left(t-\tau_{\mathrm{ref}}\right)^{2}+\omega_{0}\left(t-\tau_{\mathrm{ref}}\right)+\phi_{0}\right)\right] \\
& \quad \times \exp \left[-\alpha l_{\text {ref }} / 2\right]
\end{aligned}
$$

where $\eta_{I L}$ is a constant that depends on the insertion loss of the coupler, $\alpha$ is the fiber power attenuation coefficient in $\mathrm{m}^{-1}$ and $l_{\text {ref }}$ is the path-length of the reference signal in $\mathrm{m}$. For simplicity, the reflectivity of the reference mirror is assumed to be $100 \%$.

The electrical fields of the test signals arriving at the detector can be expressed as

$$
\begin{aligned}
E_{i}(t)= & \eta_{I L} E\left(t-\tau_{i}\right) r_{i}\left[\omega\left(t-\tau_{i}\right)\right] \exp \left[-\alpha l_{i} / 2\right] \\
= & \eta_{I L} E_{0} \exp \left[\mathrm{j}\left(\pi \gamma_{\nu}\left(t-\tau_{i}\right)^{2}+\omega_{0}\left(t-\tau_{i}\right)+\phi_{0 i}\right)\right] \\
& \times r_{i}\left[\omega\left(t-\tau_{i}\right)\right] \exp \left[-\alpha l_{i} / 2\right],
\end{aligned}
$$

where the function $r_{i}[\omega(t)]$ is the complex amplitudereflection factor of the FBG $\# i$. This reflection factor is a function of optical frequency, $\omega$, and can be mapped from optical frequency scale to time scale under the condition that frequency of the laser source is swept linearly in time as schematically represented in figure 2.

Amplitude reflection factor of each FBG can be represented by its amplitude, $a_{i}[\omega(t)]$, and its phase $\Phi_{i}[\omega(t)]$ as

$$
r_{i}[\omega(t)]=a_{i}[\omega(t)] \exp [\mathrm{j}(\Phi(\omega(t))]
$$

The reflected light, $E_{i}(t)$, from each FBG in the concatenation, interferes with the reference light from the reference mirror, $E_{\text {ref }}(t)$. The global effect is the generation of a composite signal at the photodetector which includes the sum of the responses from all the FBGs and can be analyzed 


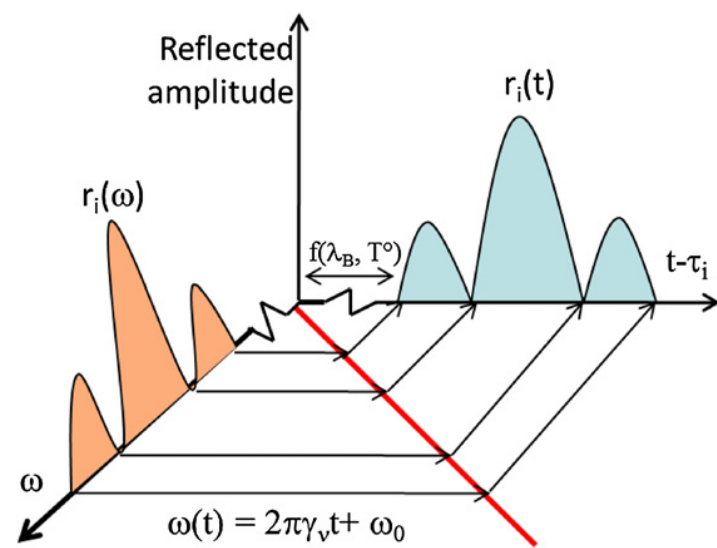

Figure 2. Amplitude reflection coefficient is a function of optical frequency and can also be represented as a function time as the frequency of the TLS is swept linearly in time ( $\gamma_{v}$ constant $)$.

by using the two-beam interference method. The global signal detected at the photodiode is given by

$$
u(t)=\sigma\left[E_{\mathrm{ref}}(t)+\sum_{i=1}^{N} E_{i}(t)\right]^{2},
$$

where $\sigma$ is a constant that depends on the photodetector sensitivity and $N$ is the number of FBGs in the concatenation. The ac part of the interference signal detected at the photodiode can be expressed as

$$
\begin{aligned}
u(t) & \propto 2 \sigma E_{0}^{2} \sum_{i=1}^{N} \kappa_{i} a_{i}[\omega(t)] \cos \left[2 \pi \gamma_{\nu}\left(\tau_{i}-\tau_{\text {ref }}\right) t\right. \\
& \left.+\Phi(\omega(t))+\varphi_{i}\right],
\end{aligned}
$$

where $\kappa_{i}$ is a constant representing all the fiber attenuation and coupling losses for the FBG \# $i, \varphi_{i}$ is a constant (which is a function of $\left.\omega_{0}, \phi_{0}, \tau_{\text {ref }}, \tau_{i}\right)$.

Distortion occurs when a concatenation of gratings sharing the same spectral characteristics are addressed simultaneously due to light having to pass through all the FBGs located between the FBG under test and the interrogator (spectral-shadowing crosstalk). This problem, which is analyzed elsewhere [15], has not been taken into account in equation (13).

It can be seen from equation (13) that the amplitude of the reflection spectrum of each FBG, $a_{i}[\omega(t)]$, modulates a sinusoidal function with a unique beat frequency $\left(f_{b i}=\right.$ $\left.\gamma_{\nu}\left(\tau_{i}-\tau_{\text {ref }}\right)\right)$. Therefore, when Fourier transform of equation (13) is taken to obtain the OFDR trace, discrete reflections of this trace are related to the Fourier transforms of the FBG reflection spectra.

If a temperature change is applied on one of the FBGs in the concatenation, for instance on the FBG $\# N$, the reflection spectrum of the FBG $\# N$ will be shifted by $\Delta \lambda$. Hence, $r_{N}[\omega(t)]$ will become $r_{N}[\omega(t)-\Delta \omega]$. As the laser frequency is linearly swept, this temperature-induced spectrum shift $\triangle \omega$, $\left(\Delta \omega=2 \pi c \frac{\Delta \lambda}{\lambda^{2}}\right)$, can be expressed in terms of an equivalent time shift, $\tau_{\text {DUT }}$ which is given by

$$
\tau_{\text {DUT }}=c \frac{\Delta \lambda}{\lambda^{2}} \gamma_{v}^{-1} \text {. }
$$

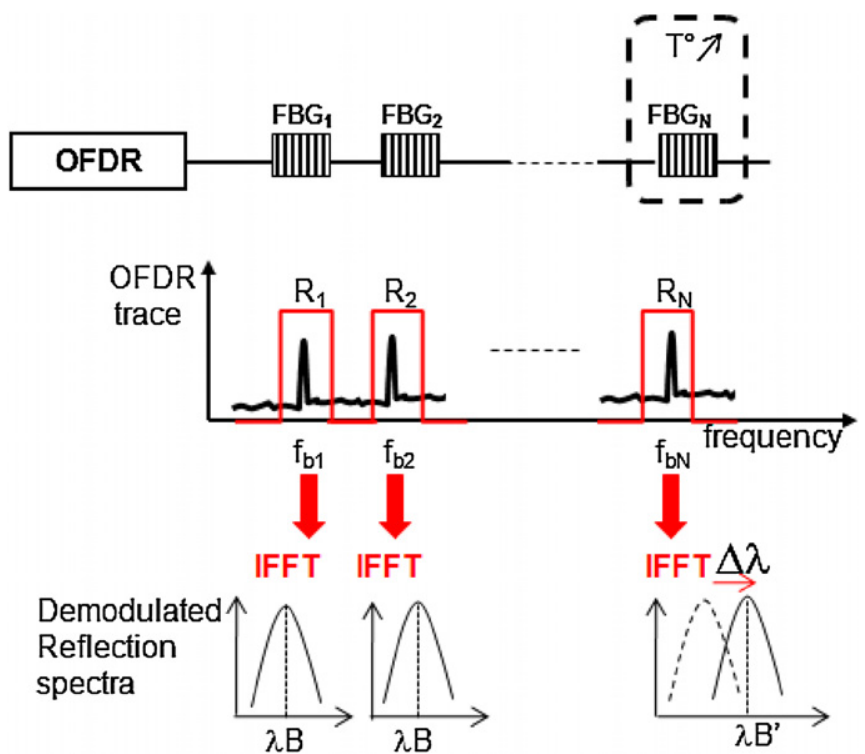

Figure 3. Schematic of the sensor principle.

As stated before, Fourier transform of the reflection spectrum of FBG \#N is found around the beat frequency $f_{\mathrm{bN}}$, $\left(f_{\mathrm{bN}}=\gamma_{\nu}\left(\tau_{N}-\tau_{\mathrm{ref}}\right)\right)$. Due to the time-delay property of Fourier analysis, the amount of shift in the time domain $\left(\tau_{D U T}\right)$ is equivalent to a shift in the phase slope in the frequency domain, i.e. $r_{i}\left(t-\tau_{\text {DUT }}\right) \leftrightarrow R_{i}(f) \exp \left(-2 \pi \mathrm{j} f \tau_{\text {DUT }}\right)$ and the reflection spectrum of each FBG can be obtained by bandpass filtering the signal in the frequency domain around each beat frequency with a sufficient bandwidth. Then, inverse fast Fourier transform (IFFT) on this selected portion can be used to recover the complex reflection spectrum of each grating independently from the others [13].

One should note that, after IFFT process, reflection coefficients of the FBGs can be mapped from time scale to the optical frequency scale as schematically represented in figure 2. Finally, by using the calibration characteristics, the temperature is deduced for each FBG in the network.

These steps are depicted in figure 3 which summarizes the sensor principle. OFDR interrogates a concatenation of $N$ identical low-reflectivity FBGs with identical Bragg wavelength (termed $\lambda_{B}$ in the figure). As schematically represented in this figure, if grating $N$ is subject to a temperature increase whereas all the others are in identical temperature conditions (room temperature), then the Bragg wavelength of the $N$ th grating is shifted by $\Delta \lambda$. First of all, the composite OFDR signal detected in time domain is converted into frequency domain (called OFDR trace in figure 3). Then, the reflection spectrum of each grating in the array is extracted by using the OFDR trace and signal processing steps (band-pass filtering and IFFT) as explained previously. Finally, from the estimation of their Bragg wavelength and from their temperature characteristic individually established in advance in a calibration process, the temperature is deduced for each FBG in the array. Note that, when a sufficient wavelength range is swept by the TLS inside the OFDR, analysis of only one OFDR trace is enough to interrogate the temperature profile of the whole array. The 


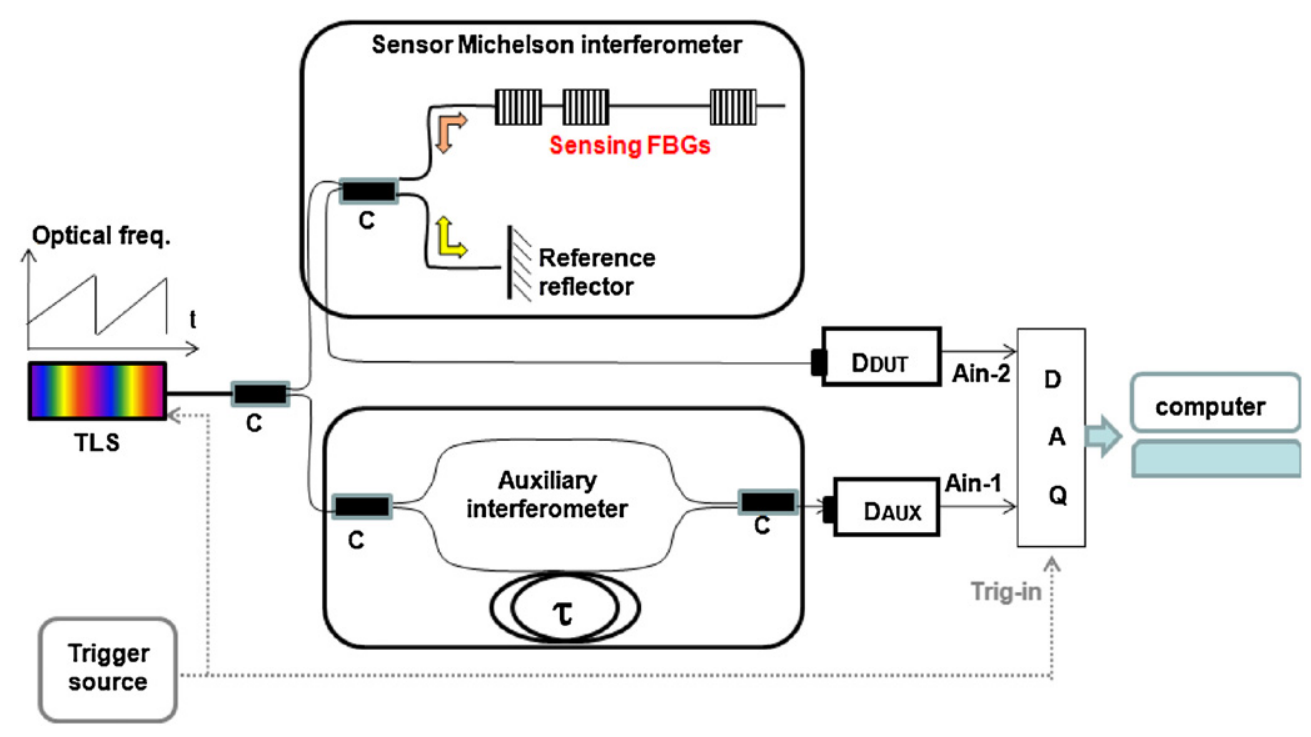

Figure 4. System diagram of the sensor demonstration (TLS: tunable laser source, D: detector, C: coupler, DAQ: data acquisition card).

optical wavelength range to be swept depends also on the temperature range to be measured.

\section{Experimental set-up and results}

\subsection{Experimental set-up}

A demonstration of the sensor using a concatenation of ten FBGs was realized. The layout of the experimental OFDR system is shown in figure 4. It comprises both the main (or test) interferometer including the FBGs to be tested and an auxiliary interferometer. The latter is used to compensate the effect of nonlinear frequency sweep to get a spatial resolution in the millimeter range [14]. For a given measurement, the outputs of the auxiliary and the main interferometers are acquired simultaneously on two analog input channels (Ain-1 and Ain2 in figure 4) via the data acquisition card (DAQ) and signal processing is realized by the computer. The operations of the DAQ and the TLS are triggered by an external TTL trigger source.

The gratings used in the sensor were manufactured with hydrogenated single mode fiber (Corning SMF 28) in the clean room facilities of UMONS. Re-coating was not operated after inscription. The FBG has the following parameters: $L=1 \mathrm{~cm} ; \Lambda=547 \mathrm{~nm}$, about $10 \%$ of reflectivity. During measurements, gratings are placed into a temperature-controlled environment which is made up of a temperature-regulated arrangement of long (about $10 \mathrm{~cm}$ ) and thin (diameter of a few $\mathrm{mm}$ ) cylinders. A stable (within $0.1^{\circ} \mathrm{C}$ ) and uniform temperature along all FBGs is therefore ensured.

In the set-up, the TLS is a commercial external cavity laser (ECL) whose nominal tuning rate is set to about $\gamma_{v}=$ $3 \mathrm{THz} \mathrm{s}^{-1}$ (about $2.5 \mathrm{~nm}$ sweep span over $100 \mathrm{~ms}$ sweep time). The chosen optical power of the TLS is $0.1 \mathrm{~mW}$. The tuning range of $2.5 \mathrm{~nm}$ corresponds to a $250{ }^{\circ} \mathrm{C}$ temperature range.

For the temperature sensor application realized in this paper, a good spatial resolution is of paramount importance to be able to properly demodulate the FBGs. It is therefore of interest to observe the OFDR trace corresponding to the FBGs by using the algorithm to suppress the nonlinearities in the optical frequency sweep. This method was reported in [14].

The OFDR spectra obtained with and without optical frequency linearization are presented in figure 5 (for a single FBG in the concatenation). The reflection peak corresponding to the FBG is broadened over $10 \mathrm{~cm}$ because of nonlinear optical frequency, whereas the OFDR spectrum becomes well limited to about $1 \mathrm{~cm}$ after the nonlinear optical frequency compensation. This compensated spectrum is then compared with the theoretical OFDR trace in figure 5(b) and shows very good agreement with the simulation results.

The spatial resolution obtained after the linearization process becomes about $0.4 \mathrm{~cm}$, which is close to the best spatial resolution of $0.2 \mathrm{~cm}$ (the best spatial resolution is determined by the frequency resolution of the data acquisition process). Indeed, the best spatial resolution is determined by the range of optical frequency that is swept $(\Delta v)$. However, this theoretical value can be deteriorated by some effects such as the superposition of the side lobes (wings) of the sine cardinale functions in the spectral signature, linewidth of the laser source and nonlinearities in the optical frequency modulation.

Independently of these effects, let us consider the ideal case where the laser source has zero linewidth and where the optical sweep is perfectly linear. When performing the measurement in this ideal case, the spatial resolution for a given measurement range also depends on the frequency resolution provided by the data acquisition system which in turn is related to the sampling frequency and the number of sampling points used in the FFT process. If the frequency resolution of the signal analyzer is not good enough, the theoretical resolution cannot be observed. In this case, for the signal analyzer with a given frequency resolution, $\Delta f$, the spatial resolution is given by

$$
\Delta z=\frac{c}{2 n_{g} \gamma_{\nu}} \Delta f
$$



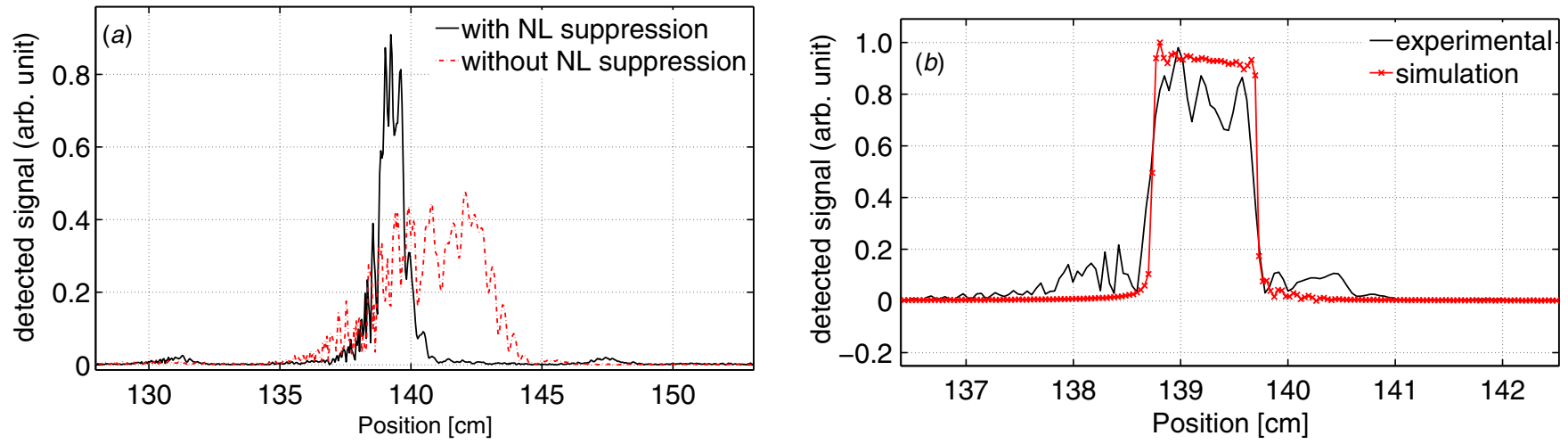

Figure 5. (a) Measured beat spectrum of the main interferometer having an FBG in the DUT without (red dashed line) and with (black solid line) the nonlinear optical frequency suppression, $(b)$ simulated OFDR spectrum for the ideal case of perfectly linear optical frequency tuning (red-crosses) and the experimental OFDR spectrum with the nonlinear optical frequency suppression (black solid line).

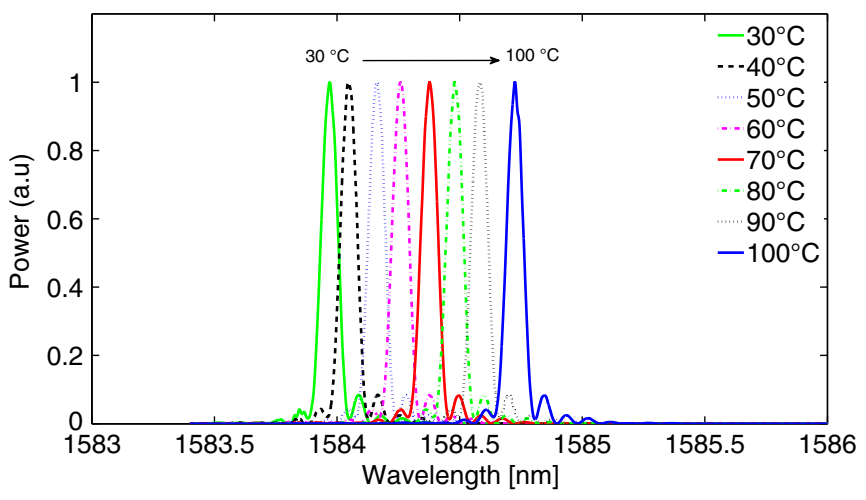

Figure 6. Evolution of Bragg grating reflected spectrum as a function of temperature (temperature increasing from left to right, from $30{ }^{\circ} \mathrm{C}$ to $100{ }^{\circ} \mathrm{C}$ in steps of $10^{\circ} \mathrm{C}$ ).

where $c$ is the speed of light in vacuum, $n_{g}$ is the refractive group index of the DUT. In this equation, the frequency sweep is assumed to be entirely linear with constant tuning rate $\left(\gamma_{\nu}\right)$.

\subsection{Characterization stage}

We first determined the dependence of the Bragg wavelength on temperature for the concatenated gratings by using our sensor system. Figure 6 shows the experimental evolution of the reflected spectrum as a function of temperature obtained by our interrogation system comprising C-OFDR and a single FBG. The shift of the Bragg wavelength is clearly seen whereas no modification is observed in the shape of the reflection spectrum.

Figure 7 represents the experimental evolution of the Bragg wavelength for the same grating as a function of temperature. The slope computed on the linear fit (or grating sensitivity) of the evolution is equal to $10.07 \mathrm{pm} /{ }^{\circ} \mathrm{C}$

\subsection{Temperature demodulation}

When the reflection spectrum of a grating is obtained, the useful information is on the main (or central) lobe. We used the maximum value data point on the main lobe to determine the Bragg wavelength. The wavelength spacing on the reflection

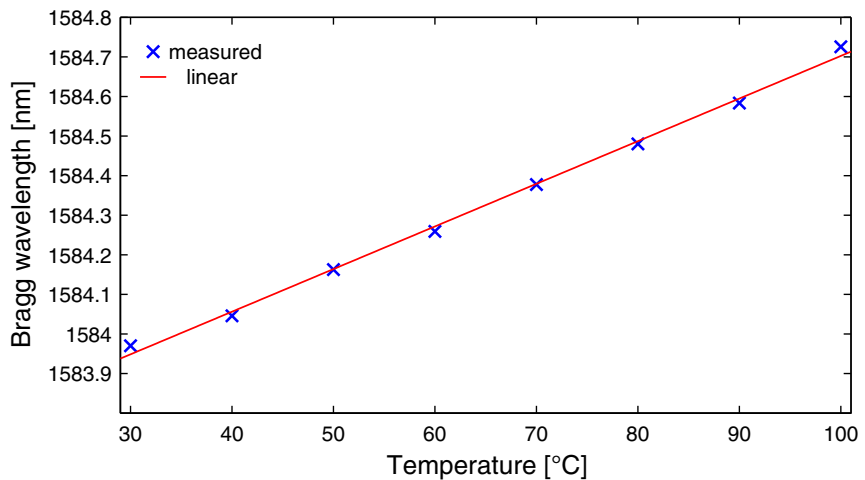

Figure 7. Evolution of Bragg wavelength as a function of temperature.

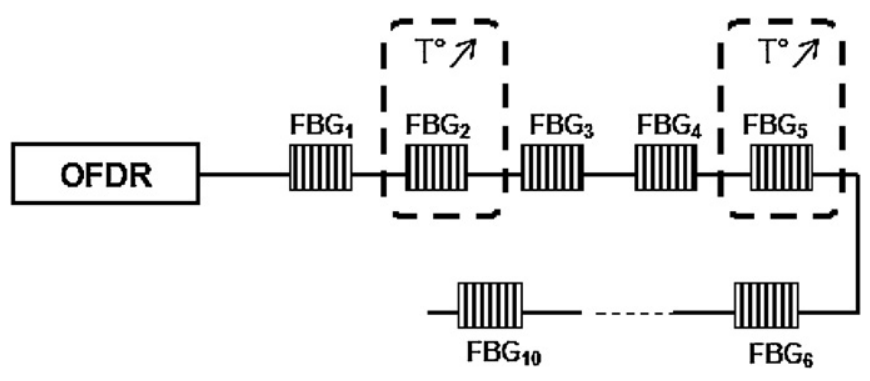

Figure 8. Schematic of the experiment.

spectrum obtained by our interrogation system is about $0.1 \mathrm{pm}$ which corresponds to a temperature resolution of $0.01{ }^{\circ} \mathrm{C}$.

Once we find the Bragg wavelength, the corresponding temperature is then determined using the reference characteristics previously measured for each grating.

\subsection{Tested sensor configuration}

A non-uniform temperature profile was imposed on ten concatenated FBGs as illustrated in figure 8. The distance between successive FBGs is about $20 \mathrm{~cm}$. All the sensing points are identical uniform FBGs (same Bragg wavelengtharound $1583.7 \mathrm{~nm}$ at room temperature-and reflectivity below 10\%). FBG2 and FBG5 were placed in the oven and 

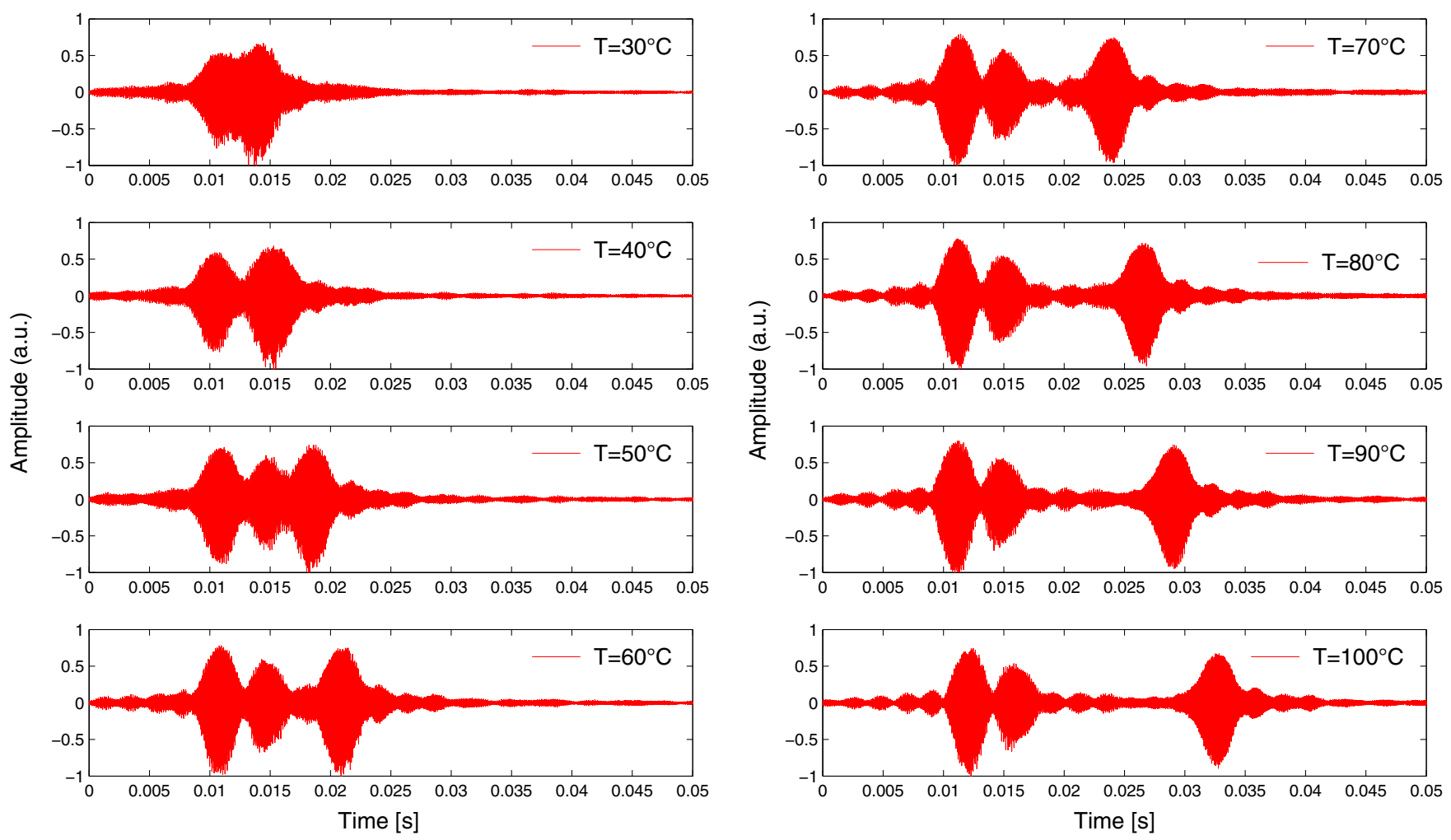

Figure 9. OFDR signal in time domain for different temperature values applied on FBG \# 2 and \# 5 .

simultaneously exposed to temperature shifts (within $0.1{ }^{\circ} \mathrm{C}$ uncertainty) between room temperature and $100{ }^{\circ} \mathrm{C}$ with a $10{ }^{\circ} \mathrm{C}$ step. The remaining eight gratings stay in a constant environment (room temperature).

\subsection{Measurement results}

The composite OFDR signals measured in time domain for different temperature values are shown in figure 9. The reflection spectra of FBGs 2 and 5 are moving away with the increased temperature. One should note that spectra of other FBGs (remaining at constant room temperature) are not perfectly identical but rather show small differences in the central (Bragg) wavelength. This inevitable fluctuation is due to the fabrication process in our clean room facilities.

OFDR trace (Fourier transform of the signal measured in time domain) is shown in figure 10. In the frequency domain (converted into distance scale in the figure), the reflection peaks corresponding to all the FBGs are clearly distinguished. In order to recover the reflection spectrum of each FBG in the array, the extracted peak by the way of a band-pass filter is inverse Fourier transformed. From this, the changes in the Bragg wavelength are recorded for each FBG.

Figures 11(a) and $(b)$ represent the evolution of the sensor deduced temperature versus the reference temperature applied by the oven, for the $100{ }^{\circ} \mathrm{C}$ temperature variation. A good agreement between the reference and measured values is observed. The maximum error on the measured temperatures was of about $2{ }^{\circ} \mathrm{C}$.

Demodulated Bragg wavelength values obtained for the FBGs outside the oven stay identical over the applied

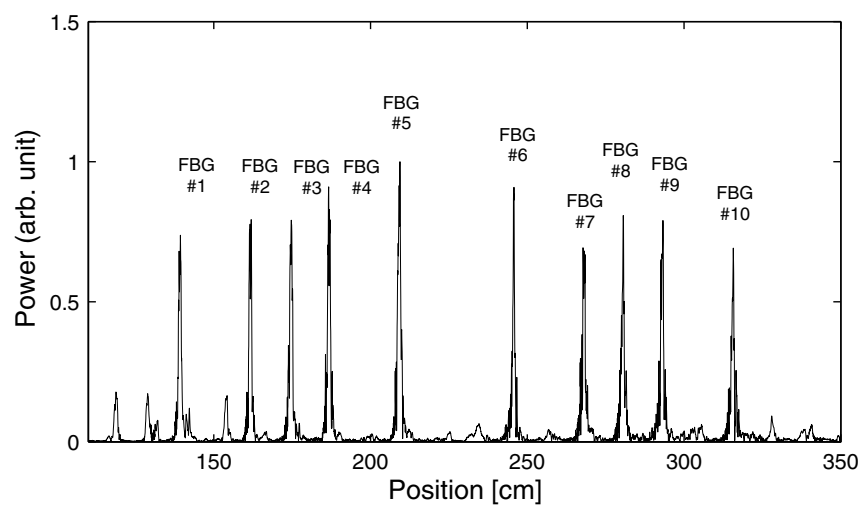

Figure 10. OFDR trace in frequency domain (converted into position).

temperature range with only slight fluctuations whose standard deviations (STD) are given in table 1. Results show that there is only a small change (smaller than $1.5^{\circ} \mathrm{C}$ ) on these FBGs due to the noise of the devices. This measurement successfully demonstrates the quasi-distributed property of the sensor.

In the last part of the measurements, for a given FBG under test and a given applied temperature, the measurements were repeated ten times. These repeatability measurements highlighted a standard deviation on the demodulated temperature smaller than $1.5^{\circ} \mathrm{C}$.

In addition to the experimental work, a study by the way of simulations was undertaken in order to get a better insight into the precision performances of the reported sensor. This simulation work was intended to study the impact of 

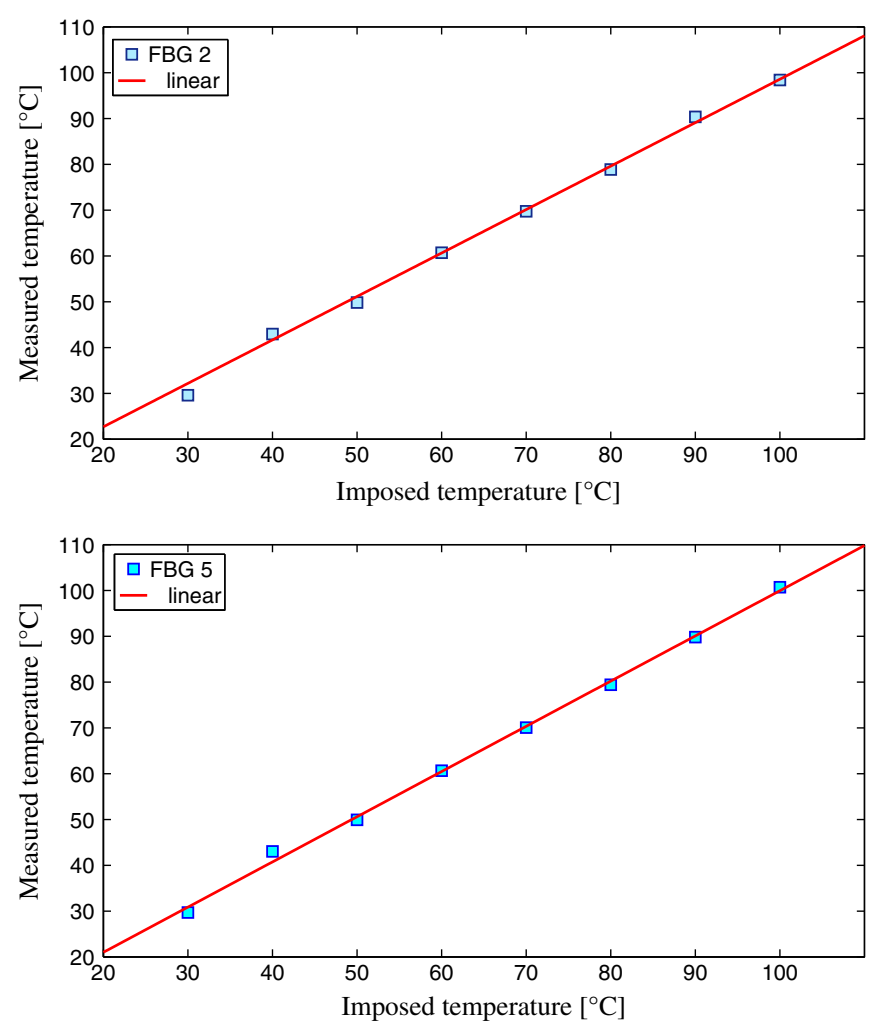

Figure 11. Evolution of the sensor-deduced temperature versus the reference temperature for FBG2 $(a)$ and FBG5 $(b)$.

Table 1. Stability measurements on the FBGs staying at room temperature.

\begin{tabular}{lll}
\hline FBG & $\mathrm{SD}(\mathrm{pm})$ & $\mathrm{SD}\left({ }^{\circ} \mathrm{C}\right)$ \\
\hline FBG1 & 14.5 & 1.45 \\
FBG3 & 14.9 & 1.49 \\
FBG4 & 12.7 & 1.27 \\
FBG6 & 12.9 & 1.29 \\
FBG7 & 13.8 & 1.38 \\
FBG8 & 13.5 & 1.35 \\
FBG9 & 11.7 & 1.17 \\
FBG7 & 13.8 & 1.38 \\
\hline
\end{tabular}

the possible shadowing crosstalk between gratings having superimposed spectra. Indeed, in the proposed sensor, the signal entered into the concatenation gets modified during its round-trip propagation along the fiber [16]. This is due to the fact that the spectrum of the light in front of each grating depends on the previous ones as their transmission spectra are superimposed. As a result, deformations in the measured grating spectrum occur. Because of the shape deterioration of the reflection spectrum, determination of Bragg wavelength, the basis of temperature measurement, becomes difficult in the presence of the non-negligible spectral-shadowing crosstalk effect.

In the simulations, all FBGs are assumed to be at room temperature and to have about identical spectral characteristics (Bragg wavelength around $1583.7 \mathrm{~nm}$ at room temperature and reflectivity below 10\%) with small fluctuations in the central (Bragg) wavelength. These inevitable fluctuations due to fabrication process correspond to the specifications of the real
Table 2. Precision analysis: mean value and standard deviation on the demodulated temperature data for the simulation of $N$ concatenated gratings subject to 1000 random temperature configurations. FBG under test $(\# N)$ is at $40{ }^{\circ} \mathrm{C}$.

\begin{tabular}{lll}
\hline $\begin{array}{l}\text { Number of } \\
\text { concatenated gratings }\end{array}$ & $\begin{array}{l}\text { Mean value of the error } \\
(\mathrm{pm})\end{array}$ & $\begin{array}{l}\text { Standard deviation } \\
(\mathrm{pm})\end{array}$ \\
\hline 10 & 3.25 & 0.62 \\
30 & 7.34 & 1.13 \\
50 & 9.79 & 1.49 \\
70 & 11.71 & 1.67 \\
100 & 14.23 & 1.87 \\
\hline
\end{tabular}

gratings written in UMONS clean room facilities and can be modeled by a Gaussian distribution of the Bragg wavelengths with a standard deviation of $50 \mathrm{pm}$. In the simulations, $22{ }^{\circ} \mathrm{C}$ corresponds to room temperature. The difference between the measured and imposed temperature is evaluated as an error value.

The mean value and standard deviation of the error (between the imposed and the demodulated temperature) for different values of $N$ are reported in table 2 when the FBG under test (last FBG) is at $40{ }^{\circ} \mathrm{C}$. Results show that, as expected, the greater the number of concatenated gratings, the larger the error (the FBG under test is the last FBG in the concatenation). For a concatenation of 100 gratings, the mean error, computed on 1000 samples, is equal to $14.2 \mathrm{pm}$, which corresponds to a temperature error of about $1.4{ }^{\circ} \mathrm{C}$.

\section{Conclusion}

This paper presents a quasi-distributed temperature sensor based on the concatenation of identical, low-reflective FBGs working as sensing points. The optical frequency-domain reflectometry technique has been used as the interrogator scheme. The distance between concatenated gratings can be of the order of a few $\mathrm{cm}$. Repeatability measurements highlighted a standard deviation on the demodulated temperature of less than $1.5^{\circ} \mathrm{C}$. The different experiments realized on the FBG array showed a very good response of the prototype for a large temperature range whatever the temperature profiles imposed on the different gratings. Simulations were realized to estimate the precision when a large number of gratings is addressed taking into account the spectral-shadowing crosstalk effect. A complete demonstration of mathematical formulas which are used to obtain the temperature information is also provided.

\section{Acknowledgments}

This research was supported by Fonds de la Recherche Scientifique-Crédit aux Chercheurs (FNRS) and the Interuniversity Attraction Pole IAP 6/10 program of the Belgian Science Policy.

\section{References}

[1] Othonos and Kalli 1999 Fiber Bragg Grating Sensors in Fibre Bragg Gratings: Fundamentals and Applications in 
Telecommunications and Sensing (Boston, MA: Artech House)

[2] Lo Y-L and Xu S-H 2006 New sensing mechanisms of an optical time domain reflectometry with fiber Bragg gratings OSA Tech. Dig. TuE4

[3] Kersey A D, Davis M A and Tsai T 1996 Fiber optic Bragg grating strain sensor with direct reflectometric interrogation Proc. 11th Int. Conf. on Optical Fiber Sensors, (Sapporo, Japan: Japan Society of Applied Physics) pp 634-7

[4] Crunelle C, Caucheteur C, Wuilpart M and Mégret P 2009 Quasi-distributed temperature sensor combining fibre Bragg gratings and temporal reflectometry technique interrogation Opt. Lasers Eng. 47 415-8

[5] Crunelle C, Caucheteur C, Wuilpart M and Mégret P 2009 Original interrogation system for a quasi-distributed FBG-based temperature sensor with fast demodulation technique Sensors Actuators A 150 192-8

[6] Crunelle C, Wuilpart M, Caucheteur C and Mégret P 2009 A quasi-distributed sensor interrogated by a wavelength-sensitive optical time-domain reflectometer Meas. Sci. Technol. 20027001

[7] Liehr S, Wendt M and Krebber K 2010 Distributed strain measurements in perfluorinated polymer optical fibres using optical frequency domain reflectometry Meas. Sci. Technol. 21094023

[8] Froggat M and Moore J 1998 High-spatial-resolution distributed strain measurement in optical fiber with Rayleigh scatter Appl. Opt. 10 1735-40

[9] Childers B A, Froggatt M E, Allison S G, Moore T C, Hare D A, Batten C F and Jegley D C 2001 Use of 3000
Bragg grating sensors distributed on four eight-meter optical fibers during static load tests of a composite structure Proc. SPIE 4332 133-42

[10] Shinoda Y, Ogura I, Ono Y, Kaneko S and Higo T 2006 Fundamental experiment of multiple-point measurement for strain by fiber Bragg gratings using optical frequency sweeping Proc. Int. Joint Conf. SICE ICASE vol 4332 pp 1672-5

[11] Abdi A M, Suzuki S, Schülzgen A and Kost A R 2007 Modelling, design, fabrication, and testing of a fiber Bragg grating strain sensor array Appl. Opt. $462563-74$

[12] Yüksel K, Wuilpart M, Moeyaert V and Mégret P 2010 Novel monitoring technique for passive optical networks based on optical frequency domain reflectometry and fiber Bragg gratings J. Opt. Commun. Netw. 2 463-8

[13] Duncan R G, Childers B A, Gifford D K, Pettit D E, Hickson A W and Brown T L 2002 Distributed sensing technique for test article damage detection and monitoring Proc. SPIE 5050 367-75

[14] Yüksel K, Wuilpart M and Mégret P 2009 Analysis and suppression of nonlinear frequency modulation in an optical frequency-domain reflectometer Opt. Express $175845-51$

[15] Yüksel K, Moeyaert V, Mégret P and Wuilpart M 2011 Analysis of spectral-shadowing crosstalk in a quasi-distributed fibre sensor interrogated by optical frequency-domain reflectometer Proc. SPIE $\mathbf{8 0 8 3} 808311$

[16] Kersey A D, Davis M A, Patrick H J, LeBlanc M, Koo K P, Askins C G, Putnam M A and Friebele E J 1997 Fiber grating sensors J. Lightwave Technol. 15 1442-63 